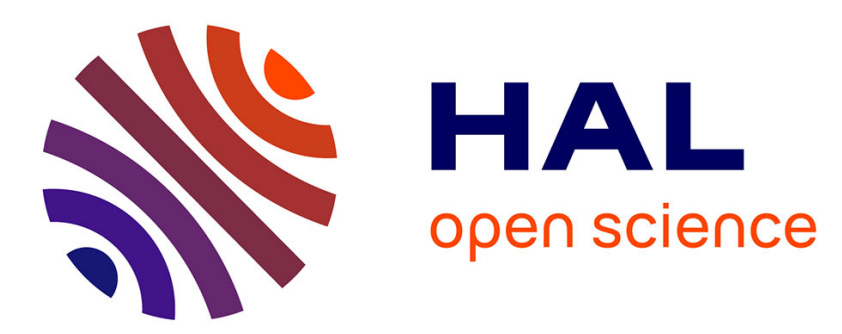

\title{
Strong spin-orbit effects in small Pt clusters: Geometric structure, magnetic isomers and anisotropy
}

P. Bloński, Samuel Dennler, J. Hafner

\section{To cite this version:}

P. Bloński, Samuel Dennler, J. Hafner. Strong spin-orbit effects in small Pt clusters: Geometric structure, magnetic isomers and anisotropy. Journal of Chemical Physics, 2011, 134 (3), pp.034107. 10.1063/1.3530799 . hal-00568227

\section{HAL Id: hal-00568227 \\ https://hal.science/hal-00568227}

Submitted on 9 Jun 2021

HAL is a multi-disciplinary open access archive for the deposit and dissemination of scientific research documents, whether they are published or not. The documents may come from teaching and research institutions in France or abroad, or from public or private research centers.
L'archive ouverte pluridisciplinaire HAL, est destinée au dépôt et à la diffusion de documents scientifiques de niveau recherche, publiés ou non, émanant des établissements d'enseignement et de recherche français ou étrangers, des laboratoires publics ou privés. 


\title{
Strong spin-orbit effects in small Pt clusters: Geometric structure, magnetic isomers and anisotropy
}

\author{
Piotr Błoński, ${ }^{1}$ Samuel Dennler, ${ }^{2}$ and Jürgen Hafner ${ }^{1, a)}$ \\ ${ }^{1}$ Fakultät für Physik and Center for Computational Materials Science, Universität Wien, Sensengasse 8/12, \\ A-1090 Wien, Austria \\ ${ }^{2}$ Laboratoire des Colloïdes, Verres et Nanomatériaux, Université de Montpellier II, F-34095 Montpellier, \\ France
}

(Received 23 September 2010; accepted 3 December 2010; published online 19 January 2011)

\begin{abstract}
$A b$ initio density functional calculations including spin-orbit coupling (SOC) have been performed for $\mathrm{Pt}_{n}, n=2-6$ clusters. The strong SOC tends to stabilize planar structures for $n=2-5$, whereas for clusters consisting of six atoms, three-dimensional structures remain preferred. SOC leads to the formation of large orbital magnetic moments and to a mixing of different spin states. Due to the spinmixing the total magnetic moment may be larger or smaller than the spin moment in the absence of SOC. Both spin and orbital moments are found to be anisotropic. Because of the strong SOC the energy differences between coexisting magnetic isomers can be comparable to or even smaller than their magnetic anisotropy energies. In this case the lowest barrier for magnetization reversal can be determined by a magnetic isomer which is different from the ground state configuration. (C) 2011 American Institute of Physics. [doi:10.1063/1.3530799]
\end{abstract}

\section{INTRODUCTION}

The current interest in magnetic nanostructures is motivated by the quest for nanoscale information storage devices and by potential applications in nanospintronics. ${ }^{1,2} \mathrm{~A}$ quantity of prime interest is the magnetic anisotropy, i.e., the dependence of the total energy of the system on the orientation of the magnetic moment. The magnetic anisotropy energy (MAE) determines the barrier to magnetization reversal due to thermal excitations; a large MAE is necessary to prevent the loss of information. The origin of the magnetic anisotropy is the coupling between the spin and orbital degrees of freedom; it is a fundamentally relativistic effect. In addition, magnetic anisotropy is critically dependent on symmetry and dimensionality; typically, the magnetic anisotropy is found to be much larger in low-symmetry nanostructures than in highly symmetric bulk materials. Prerequisites for a high magnetic anisotropy are a large spin magnetic moment and a strong spin-orbit coupling. Hence clusters or nanostructures of late $5 \mathrm{~d}$ elements possessing an intrinsic spin moment and a strong spin-orbit coupling (SOC) are potential candidates for magnetic nanostructures with a large MAE.

However, for such clusters SOC is of decisive importance not only for the MAE but also for determining the equilibrium geometric structure. For small Pt clusters Huda et al. ${ }^{3}$ have demonstrated that SOC stabilizes planar structures over the three-dimensional arrangements favored by scalar relativistic calculations, but magnetic properties have not been considered in their study. For neutral and charged Au clusters Häkkinen et al. ${ }^{4}$ reported an increased preference for planar structures compared to $\mathrm{Ag}$ and $\mathrm{Cu}$ clusters, in agreement with experimental studies on mass-selected clusters by Gilb et al. ${ }^{5}$

\footnotetext{
a) Author to whom correspondence should be addressed. Electronic mail: juergen.hafner@univie.ac.at.
}

The stabilization of planar configurations has been attributed to relativistic effects shifting the 6 s level closer to the $5 \mathrm{~d}$ state and leading to a stronger s-d hybridization.

An abundant literature (including many $a b$ initio studies) has been devoted to the geometric and magnetic properties of small metallic clusters (see, e.g., Refs. 6 and 7 and further references cited therein), but only a few ab initio calculations ${ }^{8-13}$ have attempted to determine the MAE. For the smallest clusters, transition-metal dimers, the investigations have demonstrated that the magnetic anisotropy is determined by the nature and occupation of the electronic eigenvalues close to the Fermi level. ${ }^{10-12}$ For magnetic moments perpendicular to the dimer axis, as in the absence of SOC, the eigenvalue spectrum consists of doubly degenerate $\pi_{d}$ and $\delta_{d}$ and nondegenerate $\sigma_{d}$ states. (Detailed representations of the eigenvalue spectra of dimers of $\mathrm{Pt}$ and other transition metals are given in the papers by Strandberg et al. ${ }^{10}$ and Błoński and Hafner). ${ }^{12}$ For magnetic moments aligned parallel to the dimer axis, SOC induces a splitting of the degenerate eigenstates due to the broken inversion symmetry. If the highest occupied eigenstate is doubly degenerate but occupied only by a single electron, the SOC-induced splitting of the eigenstate will stabilize an axial anisotropy. ${ }^{10,12} \mathrm{~A}$ remarkable result of these studies is also that not only the orbital moments but also the spin moments can display a substantial anisotropy if the SOC is strong enough. The formation of orbital magnetic moments and the origin of a large MAE in monowires has been discussed by Mokrousov et al. ${ }^{14}$ Smogunov et al., ${ }^{15}$ and Velev et $a l .{ }^{16}$ using similar arguments. The main difference is that the discrete eigenvalue spectrum of the dimer is replaced by a set of one-dimensional bands, with sharp van Hove singularities at the upper and lower edges. Under rotational symmetry (group $C_{\infty v}$ ), there are doubly degenerate and nondegenerate bands corresponding to the $\delta_{d}$ and $\pi_{d}$ and $\sigma_{d}$ states of the dimer, respectively. Exchange coupling and SOC induce a 
band-splitting. For axial magnetization the SOC-induced splitting is first order; for perpendicular magnetization the SOC has zero matrix elements within the doublets and a splitting occurs only near the band crossing points. For $\mathrm{Pt}$ monowires Smogunov et al. have predicted a giant MAEin this case the wire is ferromagnetic (FM) if the direction of magnetization is parallel to the wire, but the magnetic moments vanish if the magnetization direction is rotated to a perpendicular orientation. The spin-anisotropy predicted for the $4 \mathrm{~d}$ and $5 \mathrm{~d}$ dimers and the demagnetization of $\mathrm{Pt}$ monowires induced by a rotation of the magnetization direction indicate that the classical picture of the MAE based on perturbation theory, with a modest anisotropy of the orbital magnetic moments at isotropic spin moments, ${ }^{17}$ cannot hold for clusters of the heavy transition metals. The same remark applies to the description of the MAE in terms of an effective "giant-spin" Hamiltonian proposed by Canali et al. ${ }^{18}$ which is also based on the assumption of isotropic spin moments.

For larger clusters, most attempts to understand the MAE have been limited to semiempirical approaches; ${ }^{19,20}$ only a very few $a b$ initio calculations have been published to date. Kortus et al. ${ }^{8}$ calculated the MAE (defined as the difference in total energy for magnetic moments parallel and perpendicular to the symmetry axis) of 5- and 13-atom clusters of Co and $\mathrm{Fe}$ in terms of the second-order contribution from the SOC term, at a fixed geometry obtained from scalar relativistic calculations. For the five-atom clusters forming a trigonal bipyramid, MAE's of 0.1 and $0.2 \mathrm{meV} /$ atom have been reported for $\mathrm{Co}$ and $\mathrm{Fe}$ clusters, respectively. For an $\mathrm{Fe}_{13}$ cluster forming a distorted tetrahedron, an MAE of $0.27 \mathrm{meV} /$ atom was reported, while a $\mathrm{Co}_{13}$ cluster was described as magnetically isotropic. In mixed clusters the occupation of the sites with different atoms lowers the symmetry, leading to an enhanced MAE. The highest value of $0.37 \mathrm{meV} / \mathrm{atom}$ was reported for a $\mathrm{Co}_{10} \mathrm{Fe}_{3}$ cluster. Very recently Sahoo et al. ${ }^{13}$ reported $a b$ initio investigations of the MAE of 13-atom clusters of $\mathrm{Fe}$, $\mathrm{Co}$, and $\mathrm{Ni}$, concentrating on the influence of symmetry. For clusters with the structure of an ideal icosahedron, MAEs of 1.7, 0.31 , and $0.77 \mu \mathrm{eV} /$ atom, comparable to the MAE of the bulk metals, have been reported for $\mathrm{Fe}_{13}, \mathrm{Co}_{13}$, and $\mathrm{Ni}_{13}$, respectively. In this case, because of the high symmetry of the cluster, the leading contribution to the MAE is sixth-order in the SOC. A geometric distortion lowering the symmetry was found to enhance the MAE by orders of magnitude. For $\mathrm{Fe}_{13}$ an MAE of $0.32 \mathrm{meV} /$ atom was reported for a "Jahn-Tellerdistorted" cluster. However, only a restricted relaxation of the icosahedral structure describable by one-parameter models was permitted and the optimization was performed in a scalar relativistic mode, such that the possible influence of the SOC on the cluster structure was not considered. FernandezSeivane and Ferrer (hereafter referred to as FSF) ${ }^{9}$ performed $a b$ initio calculations of the geometric and magnetic structures of clusters of $\mathrm{Pd}, \mathrm{Ir}, \mathrm{Pt}$, and $\mathrm{Au}$ with up to seven atoms, including SOC. For each cluster between two and five different "seeds" for the geometric and magnetic structures of the cluster have been used to initialize the calculations, making together five to ten different initial configurations per cluster. The MAE was defined as the energy difference between the two solutions with the lowest energies-evidently this energy difference may or may not correspond to the true magnetic anisotropy energy, as illustrated by their results for $\mathrm{Pd}_{n}$ and $\mathrm{Pt}_{n}$. For the dimers, axial anisotropy for $\mathrm{Pt}_{2}$ and perpendicular anisotropy for $\mathrm{Pd}_{2}$ were reported, in semiquantitative agreement with our results. ${ }^{12}$ For the trimers a triangular configuration with an in-plane easy axis was reported, again in partial agreement with our results to be discussed below. A $\mathrm{Pt}_{4}$ cluster forms a rhombus with an easy axis lying in the plane-in this case we have been able to find a configuration with the same geometric but a different magnetic structure and a lower energy. For a $\mathrm{Pt}_{5}$ cluster the ground state (GS) geometry reported by FSF (Ref. 9) is a tetragonal pyramid, whereas in their "first excited state" the cluster forms a trigonal bipyramid. Evidently in this case the energy difference between these states is not an MAE, but a structural energy difference (ignoring for the moment that there are also substantial differences in the spin and orbital moments of both configurations). It is important, however, to note that this structural energy difference is of the same order of magnitude with the MAEs reported for the smaller clusters. In addition, the work of Huda et al. ${ }^{3}$ has shown that the ground state structure of a $\mathrm{Pt}_{5}$ is not three-dimensional, but that a flat geometry is preferred.

These discussions illustrate the difficulties in achieving a correct relativistic description of clusters formed by heavy $5 \mathrm{~d}$ metals. For these metals exchange and SOC induce a splitting of the Kohn-Sham eigenvalues which are of comparable order of magnitude. Energy differences between structural and magnetic isomers are also comparable and can be as small as magnetic anisotropy energies. In such a case an extended exploration of geometric and magnetic configurations space is required.

In the present work we investigated the structural, electronic, and magnetic properties of $\mathrm{Pt}_{n}$ clusters with $n \leq 6$ using ab initio density-functional theory (DFT) including SOC. Planar clusters are found to be lower in energy than threedimensional arrangements up to $n=5$. The energy differences between coexisting magnetic isomers with anisotropic spin and orbital moments are of the same order of magnitude with the MAE. These results lead to a picture of the physical mechanism determining the size of the MAE of the clusters, which is fundamentally different both from that suggested by low-order perturbation theory where the MAE is determined by a modest orbital anisotropy at isotropic spin moments ${ }^{17}$ and from a description in terms of an effective giant-spin Hamiltonian such as in molecular magnets. ${ }^{10,18}$

\section{COMPUTATIONAL SETUP}

Our calculations are based on DFT as implemented in the Vienna $a b$ initio simulation package VASP, ${ }^{21,22}$ using a planewave basis and the projector-augmented wave approach ${ }^{23}$ for describing the electron-ion interaction. The semilocal PW91 functional ${ }^{24}$ in the generalized gradient approximation (GGA) and the spin-interpolation proposed by Vosko et al. ${ }^{25}$ has been used for exchange and correlation. The use of a gradientcorrected functional is essential for magnetic systems. If the local-density approximation (LDA) is used, the ground state of $\mathrm{Fe}$ is predicted to be hexagonal-close-packed and 
nonmagnetic instead of body-centered cubic and ferromagnetic; $\mathrm{Cr}$ is found to be non-magnetic instead of antiferromagnetic (AFM) in the LDA ground state. In contrast, the GGA predicts the correct structural and magnetic ground states in both cases. ${ }^{26}$ For a $\mathrm{Pt}_{2}$ dimer an extended fully relativistic investigation of the performance of different local and semilocal exchange-correlation functionals in comparison with quantum-chemical approaches was performed by Anton et al. ${ }^{27}$ It was concluded that spin-polarized GGA functionals lead to excellent agreement with the experimental bond length and binding energy. The GGA predictions are far superior to $\mathrm{LDA}$ results and for $\mathrm{Pt}_{2}$ even better than quantumchemical data.

Spin-orbit coupling has been implemented in VASP by Kresse and Lebacq. ${ }^{28}$ Following Kleinman ${ }^{29}$ and MacDonald et $a l .,{ }^{30}$ the relativistic Hamiltonian given in a basis of total angular momentum eigenstates $\left|j, m_{j}\right\rangle$ with $j=l \pm 1 / 2$ (containing all relativistic corrections up to order $\alpha^{2}$, where $\alpha$ is the fine-structure constant) is recast in the form of $2 \times 2$ matrices in spin-space by reexpressing the eigenstates of the total angular momentum in terms of a tensor product of regular angular momentum eigenstates $|l, m\rangle$ and the eigenstates of the $z$-component of the Pauli spin matrices. The relativistic effective potential consists of a term diagonal in spin-space which contains the mass-velocity and Darwin corrections and the spin-orbit operator,

$$
\mathbf{V}=\mathbf{V}^{\mathrm{sc}}+\mathbf{V}^{\mathrm{SO}}=\sum_{l, m}\left[V_{l} \cdot \mathbf{1}_{\sigma}+V_{l}^{\mathrm{SO}} \overrightarrow{\mathbf{L}} \cdot \overrightarrow{\mathbf{S}}\right]|l, m\rangle\langle l, m|,
$$

where $\mathbf{1}_{\sigma}$ is the unit operator in spin-space and

$$
\overrightarrow{\mathbf{L}} \cdot \overrightarrow{\mathbf{S}}=\frac{1}{2}\left(\begin{array}{cc}
L_{z} & L_{-} \\
L_{+} & -L_{z}
\end{array}\right) .
$$

The $l$-components of the scalar $V_{l}$ and spin-orbit $V_{l}^{\text {SO }}$ potentials are weighted averages over the $l \pm 1 / 2$ components, assuming that the magnetization is directed along the $z$-axis. The Hamiltonian is therefore a $2 \times 2$ matrix in spin-space. The nondiagonal elements arise from the spin-orbit coupling, but also from the exchange-correlation potential when the system under consideration displays a noncollinear magnetization density. Calculations including spin-orbit coupling have therefore to be performed in the noncollinear mode implemented in VASP by Hobbs et al. ${ }^{31}$ and Marsman and Hafner ${ }^{32}$ where the Hamiltonian is a functional of the $2 \times 2$ density matrix $n^{\alpha \beta}(\vec{r})$. The electron density $n(\vec{r})$ is given by the trace of the density matrix, and the density matrix may be written as a linear combination of the $2 \times 2$ unit matrix and the vector $\vec{\sigma}=\left(\sigma^{x}, \sigma^{y}, \sigma^{z}\right)$ of the three Pauli spin matrices,

$$
n^{\alpha \beta}(\vec{r})=\frac{\left[n(\vec{r}) \delta_{\alpha \beta}+\vec{m}(\vec{r}) \cdot \vec{\sigma}^{\alpha \beta}\right]}{2},
$$

with the magnetization density $\vec{m}(\vec{r})=\sum_{\alpha \beta} n^{\alpha \beta}(\vec{r}) \cdot \vec{\sigma}^{\alpha \beta}$. For a local magnetization $\vec{m}(\vec{r})$ directed along the unit vector $\vec{n}=(\sin \theta \cos \phi, \sin \theta \sin \phi, \cos \theta)$, the exchange splitting is $b(\vec{r}) \vec{n} \cdot \overrightarrow{\mathbf{S}}$ where $b(\vec{r})=\delta E_{x c}\left[n^{\alpha, \beta}(\vec{r})\right] / \delta m(\vec{r})$ is the functional derivative with respect to the spin density $m(\vec{r})=|\vec{m}(\vec{r})|$. The spin-orbit interaction is transformed using the Wigner spin-rotation matrices $D^{1 / 2}(\theta, \phi)$ to 33,34

$$
V_{l}^{\mathrm{SO}} D^{1 / 2 \dagger}(\theta, \phi) \overrightarrow{\mathbf{L}} \cdot \overrightarrow{\mathbf{S}} D^{1 / 2}(\theta, \phi) .
$$

Note that both spin-orbit coupling and noncollinearity lead to a mixing of different spin-components.

Local electronic and magnetic properties can be calculated by projecting the plane-wave components of the eigenstates onto spherical waves within atomic spheres. Within each atomic sphere, spin and orbital momentums are referred to the local spin quantization axis. The local spin and orbital moments at the individual atoms have been determined by integrating the local angular-momentum- and spin-decomposed partial densities of states over occupied states. Note that the projection of the plane-wave basis states onto atomic spheres necessarily introduces a small error in the derived local quantities.

The calculations were performed for clusters placed into the center of a large cubic box with an edge length of $12 \AA$. For larger planar clusters the edge length was increased along the longest axis of the cluster to verify that the results are not influenced by interaction of the cluster with its periodically repeated images. The basis set contained plane waves with a kinetic energy of up to $500 \mathrm{eV}$; a convergence of the total energy within $10^{-7} \mathrm{eV}$ was imposed during the self-consistency cycle. The cluster structure was relaxed without any symmetry constraints until the forces on all atoms were less than $10 \mathrm{meV} / \mathrm{A}$. For selected clusters and geometries we have verified that the results remain unchanged if a larger cutoff energy or a more stringent criterion for total energy and force convergence is imposed.

For the optimization of the cluster geometry, all possible geometries identified by earlier scalar relativistic calculations which are close enough in energy to the ground state geometry have been reoptimized in calculations including SOC self-consistently. For a simultaneous optimization of all geometric, electronic, and magnetic degrees of freedom, the magnetization vector relaxes to a direction where the total energy is stationary. In addition, configurations with a fixed arbitrary direction of the magnetization can be explored by constraining the direction of the magnetization vector by applying a penalty function. For each structural isomer the magnetic structure relaxes to a locally stable configuration related to the initial one by a steepest descent path. The symmetry of this configuration corresponds to one of the magnetic point groups that can be derived from the crystallographic point group of the cluster. However, as this magnetic structure is not necessarily the ground state, we have tested for each cluster a number of magnetic configurations, including structures breaking the magnetic point group symmetry (PGS).

\section{RESULTS}

Below we shall discuss our results for the geometries, spin and orbital magnetic moments, and the magnetic anisotropy of $\mathrm{Pt}_{n}$ clusters with $n=2-6$. It has to be admitted that even at the level of scalar relativistic calculations, the results remain controversial. The literature published before 2006 has been summarized by Futschek et al., ${ }^{7}$ and the 
new results that have appeared meanwhile ${ }^{35-38}$ have not always helped to achieve a converged result. The reasons for the divergent results are difficult to assess - they are related to different pseudopotentials and basis sets as well as different criteria for stopping the self-consistency iterations and the structural optimizations. Even plane-wave calculations using the same potential can produce different results because the criteria for energy and force convergence differ between $10^{-3}$ and $10^{-7} \mathrm{eV}$ for energies and differ also widely for the residual forces. A further limitation of most calculations published so far is that static relaxation calculations always lead to a structure which is related to the initial one by a steepest-descent path. Many different initial configurations have to be used-but there is no guarantee that the global minimum has been found. To circumvent this difficulty, Futschek et ll $^{7}$ have used a dynamical simulated annealing at elevated temperature, followed by slow cooling and a final static optimization.

Spin-orbit effects on the cluster geometry have been reported by Huda et al. $^{3}$ and Sebetci; ${ }^{38}$ geometry and magnetic anisotropy have been investigated only by FSF. ${ }^{9}$ We will therefore compare our results for the geometry to the recent calculations but concentrate on the confrontation of our results for the MAE with those of FSF. In this context it is important to characterize briefly their computational setup. FSF used the local-orbital pseudopotential code SIESTA, ${ }^{39}$ norm-conserving pseudopotentials, ${ }^{40}$ and an implementation of SOC based on the so-called "on-site" approximation. ${ }^{41}$ For dimers and trimers both LDA and GGA functionals have been used, but for clusters with four and more atoms only the LDA has been used. This must be kept in mind when the results are compared.

Our results for the $\mathrm{Pt}_{2}$ dimer have been published before. ${ }^{12}$ We begin our discussion with a brief recapitulation of these results, putting them into a more general context.

\section{A. $\mathrm{Pt}_{2}$}

For a $\mathrm{Pt}_{2}$ dimer the scalar relativistic DFT-GGA calculations predict a magnetic moment of $2 \mu_{B}$ and a bond length varying between $2.33 \AA$ (plane-wave based calculations) ${ }^{3,7,35,37}$ and $2.45 \AA$ (local-orbital calculations). ${ }^{9,36}$ The calculations of Sebetci ${ }^{38}$ based on a hybrid functional yield a bond length of $2.37 \AA$. The GGA result calculated in a planewave basis agrees with the measured bond length of $2.33 \AA$ (Ref. 42).

If SOC is taken into account, the bond length increases slightly to $2.35 \AA$ for axial and to $2.38 \AA$ for perpendicular magnetization. $3,7,12$ Slightly larger bond lengths of about $2.43 \AA$ have been reported by Sebetci and FSF. $\mathrm{A} \mathrm{Pt}_{2}$ dimer has an easy magnetization direction parallel to the dimer axis; the very large MAE of $23.1 \mathrm{meV} /$ atom is a consequence of the fact that the change of the magnetization direction induces a transition from a high-moment state with $M_{J}=4.5 \mu_{B}$ for axial to a low-moment state with $M_{J}=2 \mu_{B}$ for magnetization perpendicular to the dimer axis, ${ }^{12}$ parallel to a contraction of the dimer bond length from 2.38 to $2.35 \AA$. The loss of magnetic moment arises primarily from a strong reduction of the orbital moment from $M_{L} \sim 2.7 \mu_{B}$ to $\sim 0.8 \mu_{B}$. The spin and orbital moments for the easy axis orientation are in semiquantitative agreement with FSF, who report, however, smaller spin and orbital anisotropies at a larger MAE of $37.5 \mathrm{meV} / \mathrm{atom}$.

The strong spin and orbital anisotropy calculated for the Pt dimer corresponds to the results of Smogunovet al. ${ }^{15}$ who found strained monoatomic wires of $\mathrm{Pt}$ to be FM if magnetized along the wire axis, but nonmagnetic if the magnetization direction is constrained to a perpendicular direction, resulting in a "giant" MAE of $36 \mathrm{meV} / \mathrm{atom}$. Dimer and monowires are thus first examples to demonstrate that in nanostructures with a strong SOC, a reorientation of the magnetization direction may not only change the orbital moments but also induce a transition between high- and low-moment states or even cause the total disappearance of magnetism.

\section{B. $\mathrm{Pt}_{3}$}

For the $\mathrm{Pt}_{3}$ trimer all scalar relativistic calculations agree on a structure forming an equilateral or nearly equilateral triangle, but with bond lengths varying between $2.48-2.49 \AA$ in the plane-wave calculations $\mathrm{s}^{3,7,35}$ and 2.53 $2.57 \AA$ in the local-orbital approaches. ${ }^{9,36,43}$ The magnetic structure has been described as either nonmagnetic ${ }^{35,36}$ or magnetic $^{7,9,43,44}$ with a spin moment of $2 \mu_{B}$. Futschek et al. ${ }^{7}$ report an energy difference of only $14 \mathrm{meV} /$ atom between the two magnetic isomers. Nonrelativistic quantum-chemical calculations ${ }^{46}$ predict an isocele triangle with only a very small difference in the bond lengths (2.551 and $2.543 \AA$ ) and a nonmagnetic ground state.

SOC favors a Jahn-Teller distortion to an isocele triangle with two edges measuring 2.50 and one $2.51 \AA$, point group symmetry $2 \mathrm{~mm}$ in the Hermann-Mauguin notation. The easy axis is in-plane, coincident with the mirror-plane of the structure, see Fig. 1(a). The total magnetic moment is $M_{J}$ $=2.5 \mu_{B}$, a reduced spin moment of $1.5 \mu_{B}$ due to the mixing of different spin-states is overcompensated by a collinear total orbital moment of about $1 \mu_{B}$. A mixing of singlet and triplet states, with a dominant contribution from the triplet states, is also predicted by the quantum-chemical calculations. ${ }^{46}$ Local spin and orbital moments are noncollinear, but the total spin and orbital moments are always aligned [see Fig. 1(a)]. Inplane rotation of the magnetization direction leaves total spin and orbital cluster moments unchanged, while the local moments undergo large variations [see Fig. 1(b)]. The in-plane MAE is very modest, only $0.2 \mathrm{meV} /$ atom.

The magnetic point group is $2 m^{\prime} m^{\prime}$ (where the prime indicates that the symmetry operation must be combined with a time-reversal operation which reverses the direction of axial vectors such as the magnetic moment $)^{45}$ for the ground state and $m^{\prime} m 2^{\prime}$ for the hard in-plane direction. For perpendicular magnetization the $\mathrm{Pt}_{3}$ cluster forms an equilateral triangle; both spin and orbital magnetic moments are strongly reduced; together we find $M_{J}=1 \mu_{B}$, with an MAE of $5.1 \mathrm{meV} /$ atom relative to the in-plane easy axis configuration. The local spin and orbital moments are parallel on the site with the largest 


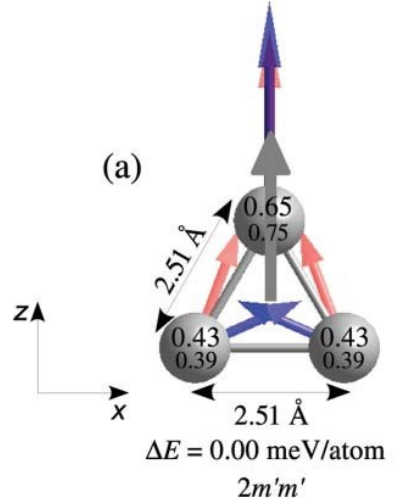

(b)

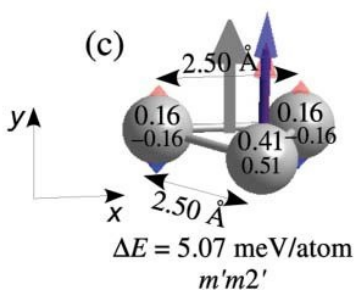

FIG. 1. Geometric and magnetic structures of a $\mathrm{Pt}_{3}$ cluster for in-plane [(a) and (b)] and perpendicular (c) magnetization (as indicated by the grey arrows). Red (light) arrows shows spin magnetic moment and blue (dark) arrows the orbital magnetic moment; the numbers give the absolute values of the local spin (upper number) and orbital (lower number) magnetic moments $M_{S}(i)$ and $M_{L}(i)$ in $\mu_{B}$. The easy axis corresponds to configuration (a), the energy for configuration (b) determines the in-plane, and (c) the axial MAE. For each configuration the magnetic point group symmetry and the energy difference relative to the ground state are listed below, cf. text.

moment, but antiparallel on the other two sites [see Fig. 1(c)]. Hence the magnetic structure breaks the threefold symmetry of the geometric arrangement; the magnetic point group symmetry is $m^{\prime} m 2^{\prime}$ as for the hard in-plane configuration. As for the dimer, rotation of the magnetization to an orientation perpendicular of the easy plane induces a transition to a different low-moment magnetic isomer.

Our result for the GS agrees reasonably well with FSF. ${ }^{9}$ However, FSF reported somewhat larger total moments of $2.96 \mu_{B}$ and $2.19 \mu_{B}$ for in-plane and perpendicular magnetization, respectively, and an MAE of $4.8 \mathrm{meV} /$ atom in the GGA, comparable to our value for the perpendicular MAE. The larger magnetic moments reported by FSF are derived from a calculation of the local spin moments using a Mulliken analysis and a calculation of the local-orbital moments via the matrix elements of the orbital moment operator in their local atomic basis set. It has been shown that the Mulliken analysis based on overlapping local orbitals systematically leads to larger values of the local moments than the integration over atomic spheres. ${ }^{13}$

\section{C. $\mathrm{Pt}_{4}$}

Scalar relativistic calculations predict for the $\mathrm{Pt}_{4}$ cluster a structure forming a slightly distorted tetrahedron with an edge length of $2.60 \AA$ and a magnetic moment of $2 \mu_{B}$, although a flat rhombus with edge length $2.50 \AA$ and moment $4 \mu_{B}$ is only $2 \mathrm{meV} /$ atom higher in energy. ${ }^{7}$ According to Sebetci, ${ }^{38} \mathrm{Nie}$ et al., ${ }^{36}$ and Xiao and Wang, ${ }^{35}$ the scalar relativistic ground state is a more strongly distorted tetrahedron with a moment of $2 \mu_{B}$, whereas Bhattacharya and Majumder ${ }^{37}$ found a highmoment rhombus with $4 \mu_{B}$ to be lower in energy than the low-moment tetrahedron by $20 \mathrm{meV} / \mathrm{atom}$. The differences in the bond length are probably due to different basis sets and more relaxed criteria for total energy and residual forces upon relaxation. A tetrahedral ground state geometry and a spin of $S=1$ is also predicted by the quantum-chemical calculations of Dai and Balasubramanian. ${ }^{47}$

Calculations including SOC predict a rhombus (point group $\mathrm{mmm}$ ) with an edge length of $2.53 \AA$, almost equal to the length of the short diagonal, to be lower in energy by $32 \mathrm{meV} /$ atom than the tetrahedron. The higher stability of the rhombus compared to the tetrahedron agrees with Huda et al. ${ }^{3}$ and FSF, ${ }^{9}$ while Sebetci ${ }^{38}$ finds the tetrahedron to be lower in energy. The local-orbital calculations predict slightly larger interatomic distances (2.55 $\AA$ according to FSF, 2.55-2.60 according to Sebetci). Surprisingly the GS is AFM with local magnetic moments $M_{J}(i)= \pm 0.54 \mu_{B}$ on the sites connected by the short diagonal, while the other two atoms are nonmagnetic due to the frustration of the AFM interactions in triangular configurations. Rotation of the AFM moments into the plane reduces both spin and orbital moments and costs an energy of $4.4 \mathrm{meV} / \mathrm{atom}$ [see Figs. 2(a) and 2(b)]. The magnetic point group symmetries of these AFM configurations is $m^{\prime} m^{\prime} m^{\prime}$.

For in-plane magnetization an FM state with spin and orbital moments aligned parallel to the short diagonal of the structure, $M_{J}=4 \mu_{B}, M_{S}=2.6 \mu_{B}$, and $M_{L}=1.4 \mu_{B}$ [see Fig. 2(c)] is energetically favored over the AFM state. This configuration corresponds to the scalar relativistic GS of the rhombus with $M_{S}=4 \mu_{B}$, with a reduced spin moment due to mixing of spin states which is just compensated by the orbital moment [Fig. 2(c)]. In-plane rotation of the FM magnetic moment to a direction parallel to the long diagonal costs an energy of $8.7 \mathrm{meV} / \mathrm{atom}$ and leads to a transition to a low-moment isomer with $M_{J}=3 \mu_{B}$ [see Fig. 2(d)]. For perpendicular magnetization we find an FM solution with $M_{J}=3.7 \mu_{B}$ with an energy intermediate between the two FM in-plane configurations [see Fig. 2(e)]. All three FM configurations have magnetic point group symmetry $\mathrm{mm}^{\prime} \mathrm{m}^{\prime}$.

Since the ground state is AFM, an MAE may be derived only for the excited FM states. The in-plane FM configuration corresponds to the ground states reported by Sebetci ${ }^{38}$ and FSF; ${ }^{9}$ we also note a quite good agreement of the total magnetic moments. FSF derived a large MAE of $12.5 \mathrm{meV} /$ atom separating in-plane and perpendicular highmoment FM states. This energy is larger than the energy difference between our FM perpendicular and in-plane configurations of $5.3 \mathrm{meV} / \mathrm{atom}$. The difference is due to the fact that FSF used the LDA for tetramers and larger clusters. For the smaller clusters FSF demonstrated that the LDA always leads to significantly larger values of the MAE.

For the three-dimensional structural isomer of $\mathrm{Pt}_{4}$, the calculations including SOC predict a perfect tetrahedral geometry (point group symmetry $\overline{4} 3 m$, edge length $2.59 \AA$ ) and a noncollinear antiferromagnetic structure [see Fig. 3(a)]. All local moments point along one of the threefold symmetry 

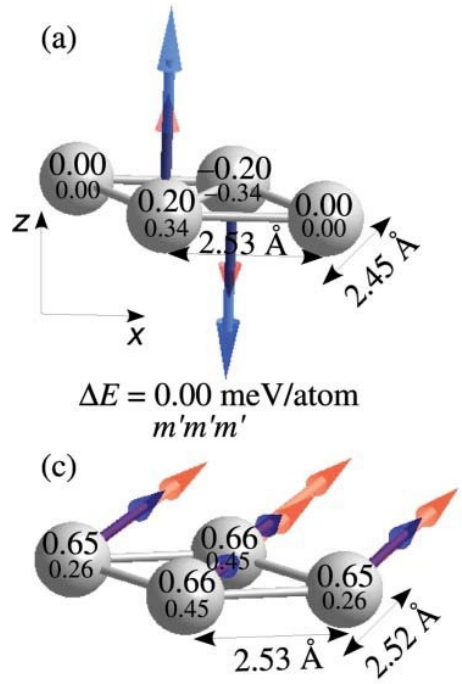

$\Delta E=2.89 \mathrm{meV} /$ atom $m m^{\prime} m^{\prime}$

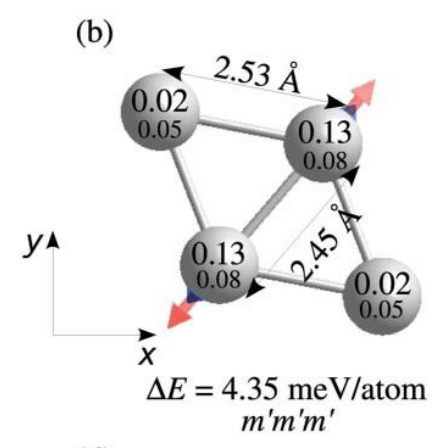

(d)

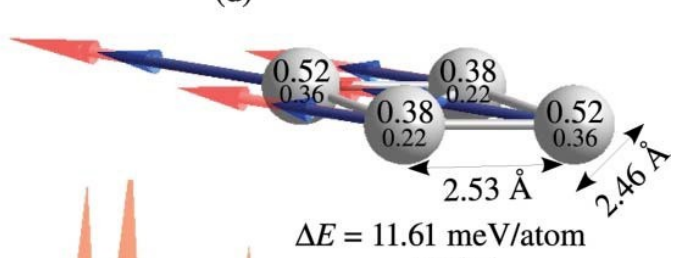

$m m^{\prime} m^{\prime}$

FIG. 2. Geometric and magnetic structures of a $\mathrm{Pt}_{4}$ cluster for in-plane [(b-d)] and perpendicular [(a) and (e)] magnetization. Configurations (a) and (b) are antiferromagnetic, and (c)-(e) ferromagnetic, cf. Fig. 1.

directions; the magnetic point group is $\overline{4}^{\prime} 3 m^{\prime}$. The noncollinear orientation of the local moments relieves the frustration of the antiferromagnetic nearest-neighbor coupling in a triangular configuration. The AFM configuration of the $\mathrm{Pt}_{4}$ tetrahedron is related not only to the AFM ground state of the planar cluster but also to the AFM ground state of the $\mathrm{Pt}_{4}$ tetrahedron with zero spin from the scalar relativistic calculations. ${ }^{7}$ However, as in these calculations noncollinearity was not considered, the frustration could be relieved only by lowering the tetrahedral symmetry. The total energy of this state is higher by $31 \mathrm{meV} /$ atom than that for the magnetic GS of the rhombus. A noncollinear magnetic GS has also been reported by FSF (Ref. 9) for a $\mathrm{Pd}_{4}$ tetrahedron.

A locally stable ferromagnetic state is found for a magnetization direction aligned parallel to one of the edges of the tetrahedron [see Fig. 3(b)]. In this configuration both local spin and orbital moments are enhanced relative to the ground state and slightly canted relative to the global magnetic moment. In this configuration the geometric structure is distorted in a way similar to that found in the scalar relativistic calculation; the magnetic point group symmetry is $2 \mathrm{~m}^{\prime} \mathrm{m}^{\prime}$. This configuration is higher in energy by $6.14 \mathrm{meV} /$ atom than the noncollinear ground state.

\section{D. $\mathrm{Pt}_{5}$}

Scalar relativistic calculations $\mathrm{s}^{3,7,35,36}$ predict that the $\mathrm{Pt}_{5}$ cluster forms a trigonal bipyramid carrying a magnetic moment of $4 \mu_{B}$, which is found to be lower in energy by $35 \mathrm{meV} /$ atom than a square pyramid with a magnetic moment of $6 \mu_{B}$. Sebetci ${ }^{38}$ finds a capped tetrahedron to

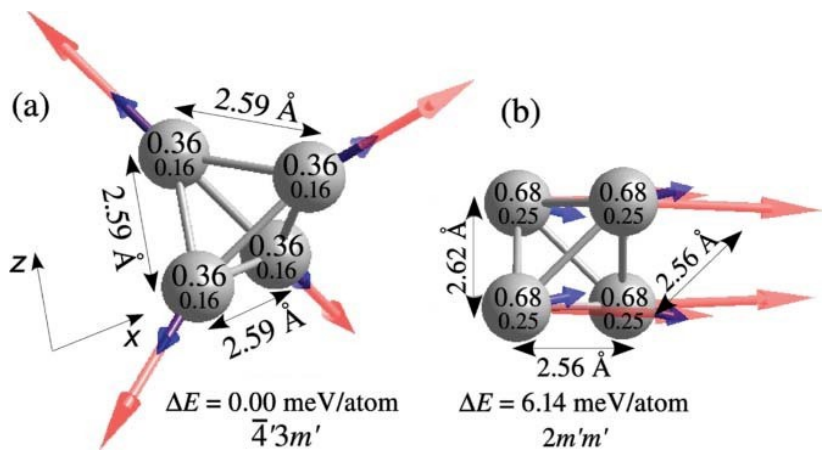

FIG. 3. (a) Noncollinear magnetic ground state structure of a $\mathrm{Pt}_{4}$ cluster with a tetrahedral geometry. (b) Ferromagnetic state with the magnetization aligned parallel to one of the edges of the tetrahedron, forming a distorted tetrahedron, cf. Fig. 1. 

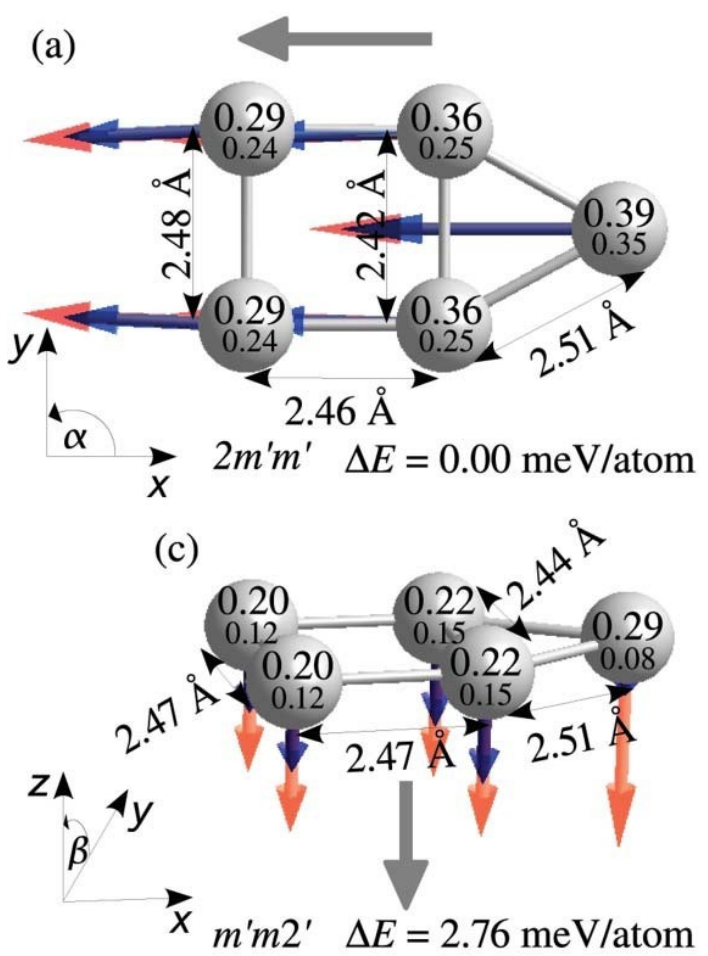

(e)

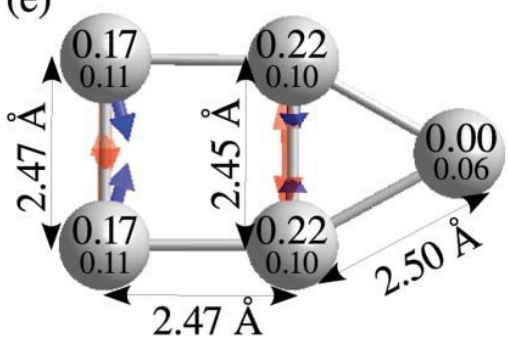

$2 m^{\prime} m^{\prime} \quad \Delta E=3.57 \mathrm{meV} /$ atom
00

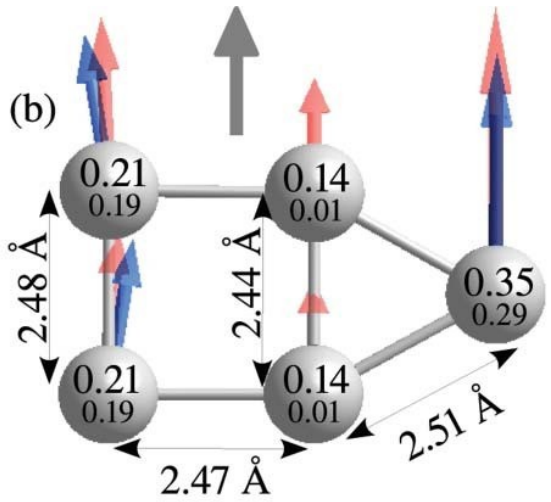

$m^{\prime} m 2^{\prime} \quad \Delta E=4.23 \mathrm{meV} / \mathrm{atom}$

(d)

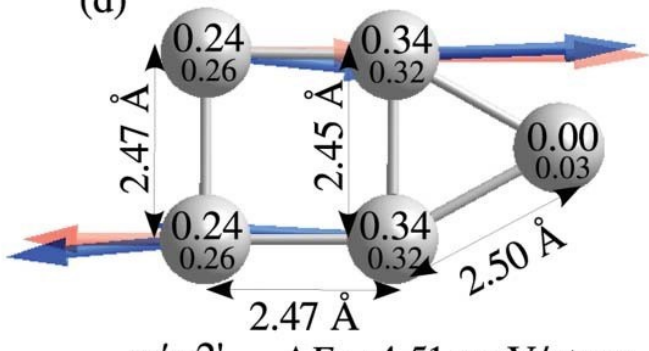

$m^{\prime} m 2^{\prime} \quad \Delta E=4.51 \mathrm{meV} / \mathrm{atom}$

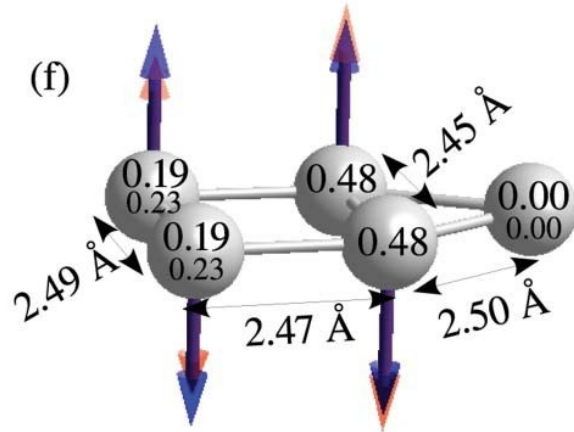

$2 \mathrm{~mm} \Delta E=1.20 \mathrm{meV} /$ atom

FIG. 4. Geometric and magnetic structures of a $\mathrm{Pt}_{5}$ cluster for perpendicular and in-plane magnetizations, cf. Fig. 1. The ground state is the high-moment configuration (a), which is energetically almost degenerate with a configuration where all magnetic moments are rotated by $180^{\circ}$. Configurations (b) and (c) are low-moment ferromagnetic and configurations (d)-(f) antiferromagnetic. The angles $\alpha$ (for in-plane rotation of the magnetization direction) and $\beta$ (for rotation to a perpendicular orientation) have been defined in the inset, cf. Fig. 5 and text.

be lower in energy, while Bhattacharya and Majumder ${ }^{37}$ find a planar cluster with a moment of $2 \mu_{B}$ to be favored already at the scalar relativistic level. A distorted trigonal bipyramid is predicted by the quantum-chemical calculations of Majumdar et al. ${ }^{46}$

If $\mathrm{SOC}$ is included, a $\mathrm{Pt}_{5}$ cluster forms a slightly distorted square plus an isocele triangle (see Fig. 4); this planar structure is favored by $40 \mathrm{meV} /$ atom over a trigonal bipyramid and by $34 \mathrm{meV} /$ atom over a square pyramid. [All structural energy differences are calculated for the magnetic ground state of the respective geometrical configurations, i.e., the FM in-plane state of the planar structure (see Fig. 4), the FM in-plane state of the square pyramid [see Fig. 6(a)], and the FM in-plane state of the trigonal bipyramid [see Fig. 7(a)]. The prediction of a planar ground state geometry agrees with the results of Huda et al., ${ }^{3}$ while Sebetci ${ }^{38}$ and FSF (Ref. 9) find the square pyramid to be the lowest in energy. Stationary magnetic states of the planar cluster for magnetization along in- and out-of-plane symmetry directions are shown in Fig. 4. In the GS [configuration (a)] with $M_{J} \sim 3 \mu_{B}\left(M_{S}\right.$ $\left.=1.7 \mu_{B}, M_{L}=1.3 \mu_{B}\right)$, the magnetization is in-plane; the total spin and orbital moments are parallel to the twofold symmetry axis of the cluster. Inversion of the direction of magnetization by $180^{\circ}$ leaves the magnetic moments and the total energy unchanged. Rotation of the direction of magnetization by $90^{\circ}$ within the plane of the cluster or to a perpendicular orientation leads to a transition to low-moments states [see Figs. 4(b) and 4(c)] which are 4.2 and $2.8 \mathrm{meV} /$ atom above the GS. For all directions of the magnetization, the FM configurations (a)-(c) coexist with AFM configurations [see Figs. 4(d)-4(f)]. 

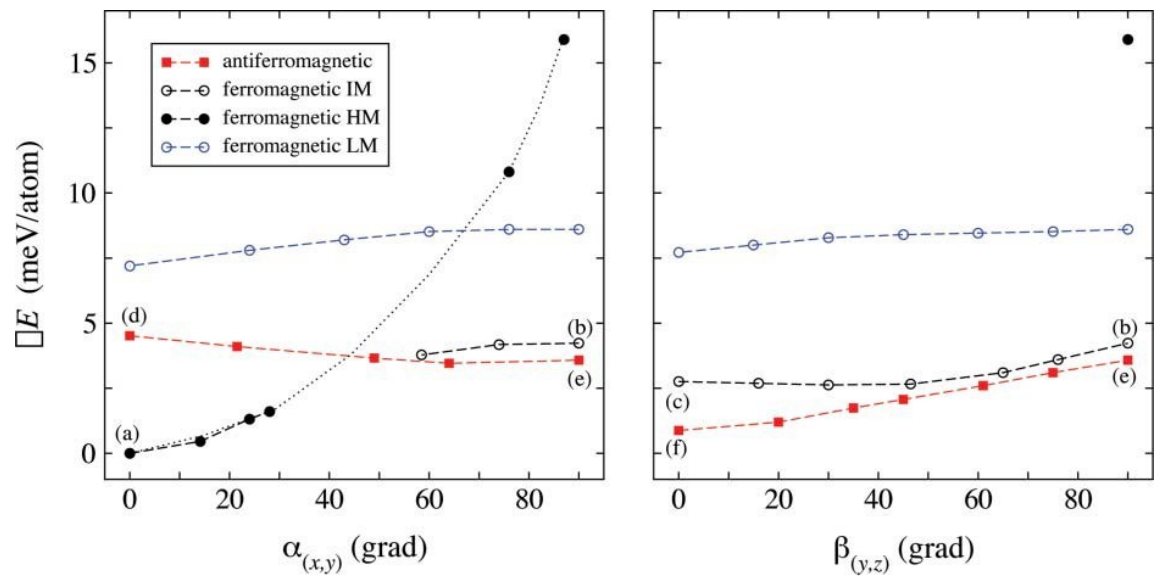

FIG. 5. Variation of the total energies of the ferromagnetic (full and empty circles) and antiferromagnetic (squares) configurations of a Pt 5 cluster upon rotation of the magnetization direction within the $(x, y)$ plane of the cluster and in the $(y, z)$ plane perpendicular to the twofold symmetry axis. See Fig. 4 for the definition of the angles. For the ferromagnetic configurations solutions with high (HM), intermediate (IM) and low (LM) magnetic moments are indicated, cf. Fig. 4.

Except for an orientation of the local magnetic moments parallel to the twofold symmetry axis [FM GS (a) and AFM configuration (d)], the AFM state is lower in energy. The lowest barrier to a magnetization reversal is via the AFM out-ofplane configuration (f), with an MAE of $1.2 \mathrm{meV} / \mathrm{atom}$. The point group symmetry of the planar $\mathrm{Pt}_{5}$ structure is $2 \mathrm{~mm}$. In the magnetic point group symmetries of the stationary configurations, one or two of the symmetry elements have to be combined with the time-reversal operation, as indicated in Fig. 4.

For all clusters the magnetic potential-energy surface as a function of the orientation of the magnetic moment consists of interpenetrating sheets representing different magnetic isomers. To explore the potential-energy surfaces in more detail, we performed for $\mathrm{Pt}_{5}$ a series of calculations with constrained orientations of the magnetic moments. If, starting from the FM high-moment ground state, we gradually rotate the magnetization direction in-plane, taking the converged charge and spin densities from the last step to initialize the self-consistency cycle, we follow a steeply ascending curve of FM high-moment states $\left(M_{J} \sim 3 \mu_{B}\right)$ ending at $\alpha=90^{\circ}$ at an energy $16 \mathrm{meV} / \mathrm{atom}$ above the GS [see Fig. 5(a)]. However, for magnetization perpendicular to the twofold symmetry axis, an FM solution with a lower moment $\left(M_{J} \sim 1.75 \mu_{B}\right)$ has an energy only $4.2 \mathrm{meV} / \mathrm{atom}$ above the GS and can also be followed to smaller angles. At somewhat higher energies of about $8 \mathrm{meV} /$ atom above the GS, we also found a lowmoment FM solution with $M_{J} \sim 1 \mu_{B}$ [see Fig. 5(a)]. At intermediate angles where the total energies of high- and lowmoment states are comparable, it is very difficult to find a well-converged solution. Coexisting FM and AFM states are also found for magnetization directions in a plane perpendicular to the twofold symmetry axis [see Fig. 5(b)]. The AFM state (f) represents the lowest barrier to magnetization reversal relative to the magnetic GS.

FSF (Ref. 9) found for the $\mathrm{Pt}_{5}$ cluster a ground state configuration in the form of a square pyramid and a huge magnetic moment of $M_{J} \sim 7 \mu_{B}$, in contrast to the present work and that of Huda et al. ${ }^{3}$ In its "first excited state" the cluster forms a trigonal bipyramid with a lower magnetic moment of $M_{J} \sim 4.4 \mu_{B}\left(M_{S}=3.6 \mu_{B}, M_{L}=0.8 \mu_{B}\right)$. Hence energy difference between these two states of $1.1 \mathrm{meV} /$ atom is not an MAE, but a structural and magnetic energy difference.

To allow a comparison with their results it is of interest to also explore the magnetic properties of the three-dimensional structural isomers of the $\mathrm{Pt}_{5}$ cluster. For the square pyramid the three stationary magnetic states we have been able to identify are shown in Fig. 6. In the magnetic GS the fourfold symmetry of the geometric structure is broken by slightly different bond lengths of 2.56 and $2.57 \AA$ in the basal plane; although all four atoms carry the same spin and orbital moments. The large total magnetic moment of $M_{J}=5.3 \mu_{B}$ $\left(M_{S}=3.9 \mu_{B}, M_{L}=1.4 \mu_{B}\right)$ is aligned parallel to the edge of the square, but the local magnetic spin and orbital moments are slightly canted relative to the overall magnetization direction [see Fig. 6(a)], in line with the reduced symmetry. Rotation of the magnetization direction parallel to the basal (a)

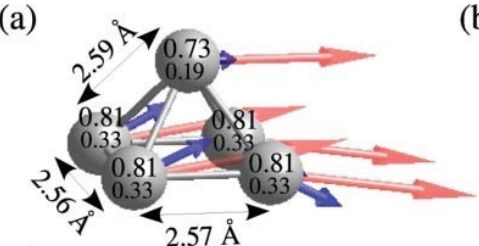

ZA

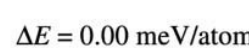
2 ' $m$ ' $m$ ' $\vec{x}$ (c)
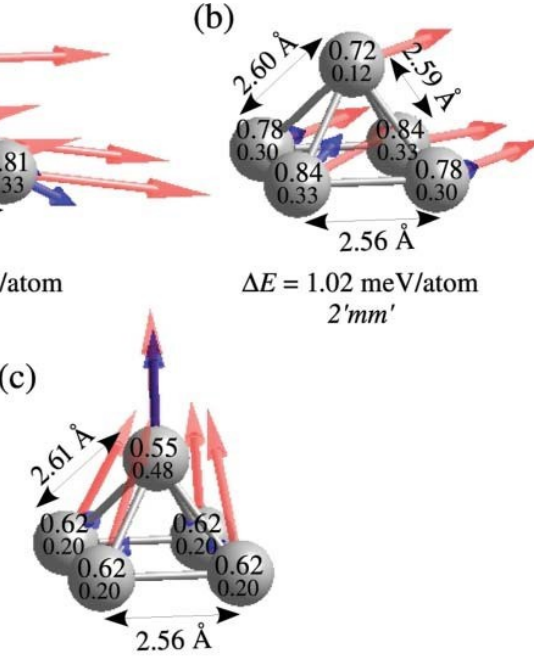

$\Delta E=1.02 \mathrm{meV} / \mathrm{atom}$ 2 ' $m m^{\prime}$
$\Delta E=20.70 \mathrm{meV} / \mathrm{atom}$ $4 m^{\prime} m^{\prime}$
FIG. 6. Geometric and magnetic structures of a $\mathrm{Pt}_{5}$ cluster forming a square pyramid for in-plane [(a) and (b)] and perpendicular (c) magnetizations, cf. Fig. 1 and text. 
(a)

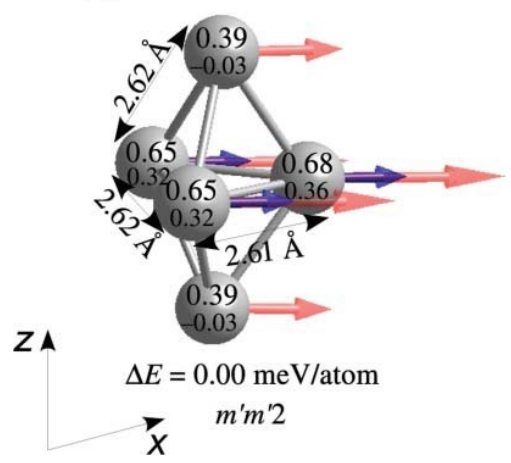

(b)

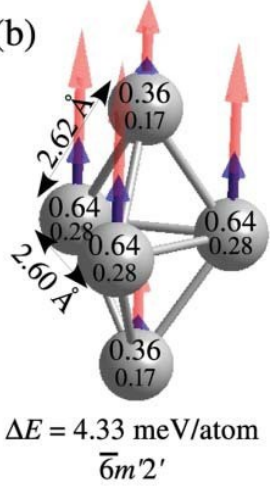

FIG. 7. Geometric and magnetic structures of a $\mathrm{Pt}_{5}$ cluster forming a trigonal bipyramid for in-plane (a) and perpendicular (b) magnetizations, cf. Fig. 1 and text.

plane requires to overcome a modest in-plane MAE of 1.02 meV/atom. In the saddle-point configuration the magnetization is parallel to the diagonal of the base; the fourfold symmetry is broken for both the geometric and magnetic structures [see Fig. 6(b)], although the total cluster moment remains unchanged. Initialization of the magnetization along the fourfold axis leads to a stationary magnetic state with a lower total moment of $M_{J}=3.9 \mu_{B}\left(M_{S}=2.9 \mu_{B}, M_{L}=\right.$ $\left.1.0 \mu_{B}\right)$ aligned along the axis, but again canted local moments [see Fig. 6(c)]. The bonds connecting the atoms in the basal plane to the vertex are slightly elongated by $0.02 \AA$. The energy of this axial low-moment state is $20.7 \mathrm{meV}$ higher than that of the ground state.

Our magnetic ground state configuration for the trigonal bipyramid is shown in Fig. 7(a). The threefold rotational symmetry is broken by a slight distortion of the central triangle. The magnetic moments are ferromagnetically aligned parallel to the central triangle of the cluster and a small difference exists between the magnetic moments of the atoms occupying its vertices. The magnetic point group symmetry is $m^{\prime} m^{\prime} 2$; the easy axis is aligned with the twofold symmetry axis. The total magnetic moment of $M_{J}=3.7 \mu_{B}$ is slightly lower than the moment calculated by FSF (Ref. 9) (considering the differences in the calculations of the local moments) and also lower than the spin moment of $4 \mu_{B}$ derived for the scalar relativistic case. In-plane rotation of the magnetic moment to a direction parallel to one of the edges of the triangle increases the energy by only $0.06 \mathrm{meV} /$ atom. Alignment of the magnetization direction parallel to the axis of the bipyramid restores the threefold symmetry axis and leads to a ferromagnetic configuration with a total moment of $M_{J}=3.8 \mu_{B}$ (magnetic point group $\overline{6} m^{\prime} 2^{\prime}$ ) which is higher in energy by $4.3 \mathrm{meV} / \mathrm{atom}$. In contrast to the strong anisotropy of both spin and orbital moments of the planar cluster, for the three-dimensional structure the spin moment is almost isotropic and the orbital anisotropy is modest on the three atoms occupying the central triangle and more pronounced only at the apices.

Hence for the two three-dimensional structural isomers of the $\mathrm{Pt}_{5}$ cluster, our analysis qualitatively confirms the observation of FSF: the out-of-plane MAE of the square pyramid of $20.7 \mathrm{meV} /$ atom is larger than the structural energy difference of $6 \mathrm{meV} /$ atom between the square pyramid and the

trigonal bipyramid in their respective magnetic ground states. However, this is a structural energy difference, and not a magnetic anisotropy energy. Also, the GS of the $\mathrm{Pt}_{5}$ cluster is planar and the MAE of the planar cluster is lower than the structural energy difference to the three-dimensional isomers.

\section{E. $\mathrm{Pt}_{6}$}

The predictions for the structure of a $\mathrm{Pt}_{6}$ cluster available in the literature scatter widely. The earlier scalar relativistic calculations in our group led to the surprising result that a capped square pyramid with a magnetic moment of $6 \mu_{B}$ is lower in energy by $23 \mathrm{meV} /$ atom than an octahedron in the same spin-state and also lower in energy than any planar configuration. ${ }^{7}$ Xiao and Wang ${ }^{35}$ found the lowest energy for a planar geometry consisting of a central square capped on two sides by two triangles, lower by about $27 \mathrm{meV} / \mathrm{atom}$ than both an octahedron and a trigonal prism. Bhattacharya and Majumder ${ }^{37}$ and Nie et al. ${ }^{36}$ predicted a planar geometry consisting of four edge-sharing triangles, which was found to be favored by a large energy difference of $110 \mathrm{meV} /$ atom over the most favorable three-dimensional structure (a capped trigonal bipyramid). Sebetci ${ }^{38}$ found a distorted trigonal prism with a moment of $6 \mu_{B}$ to be lower in energy by $25 \mathrm{meV} /$ atom than a distorted capped square pyramid with the same magnetic moment and much lower in energy than a strongly distorted octahedron. This lack of agreement on the most basic features of the cluster prompted us to repeat the scalar relativistic calculations for all candidate structures with stringent criteria on energy and force convergence. A regular trigonal prism (interatomic distances $2.57 \AA$ in the basal plane, height $2.51 \AA$ ) and a magnetic moment of $8 \mu_{B}$ were found to be the ground state with an energy lower by $11 \mathrm{meV} /$ atom than the square pyramid plus an ad-atom (magnetic moment $6 \mu_{B}$ ) and by $26 \mathrm{meV} /$ atom than an almost perfectly symmetric octahedron with a magnetic moment of $6 \mu_{B}$. Among the planar structures the configuration reported by Xiao and Wang ${ }^{35}$ was found to be favored (but higher in energy by more than $100 \mathrm{meV} /$ atom than the three-dimensional structures).

If SOC is taken into account, the energetic order is reversed in favor of the octahedron with a noncollinear lowmoment state [see Fig. 8(a)] which is found to be lower in energy by $13.8 \mathrm{meV} /$ atom than the square pyramid plus adatom with $M_{J}=5.0 \mu_{B}$ [see Fig. 8(c)]. Compared to the octahedron, a trigonal prism with a magnetic moment of $M_{J}=6.9 \mu_{B}$ [Fig. 8(e)] is energetically disfavored by $35 \mathrm{meV} /$ atom and a planar structure [similar to that of $\mathrm{Pt}_{5}$, see Fig. $8(\mathrm{~g})$ ] with $M_{J}=2 \mu_{B}$ by $140 \mathrm{meV} /$ atom. For all four structural isomers, the energy difference refers to the magnetic ground state derived from calculations with initial magnetic configurations differing in the magnitude and direction of the magnetization.

In the magnetic ground state, the $\mathrm{Pt}_{6}$ octahedron has a perfect octahedral geometry with all interatomic distances equal to $2.62 \AA$ but the distribution of the magnetic moments breaks the octahedral symmetry. The magnetic structure is noncollinear. The four $\mathrm{Pt}$ atoms occupying the equatorial plane carry spin moments of $0.26-0.27 \mu_{B}$, directed along the 
(a)

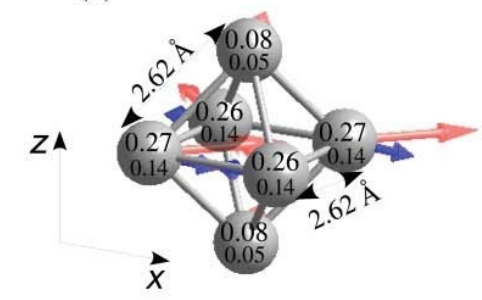

$\Delta E=0.00 \mathrm{meV} / \mathrm{atom}$

$2 ' 2$ '2

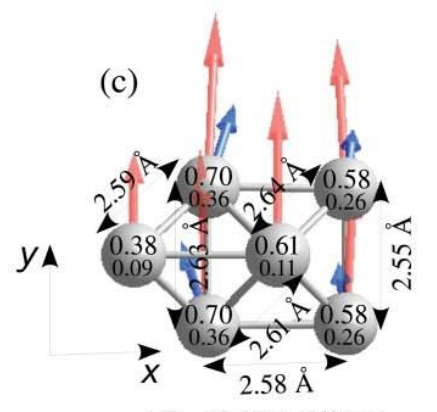

$\Delta E=13.80 \mathrm{meV} /$ atom

$m$

(e)

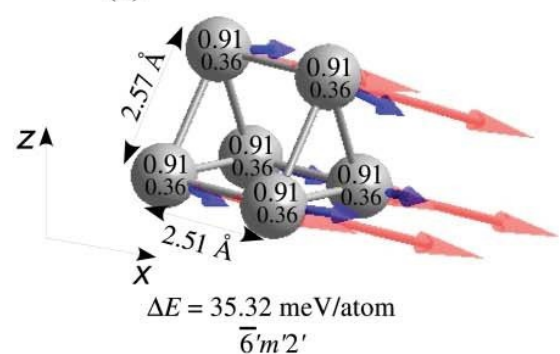

(b)

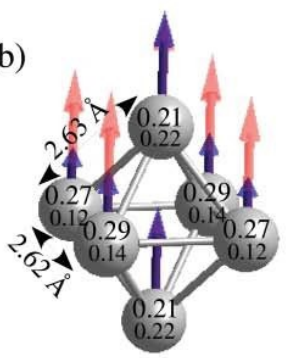

$\Delta E=1.40 \mathrm{meV} /$ atom

$2^{\prime} 2{ }^{\prime} 2$

(d)

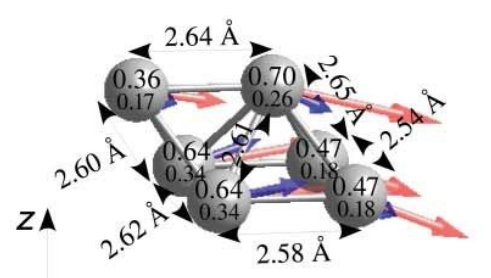

$\Delta E=16.68 \mathrm{meV} / \mathrm{atom}$

$m$

(f)

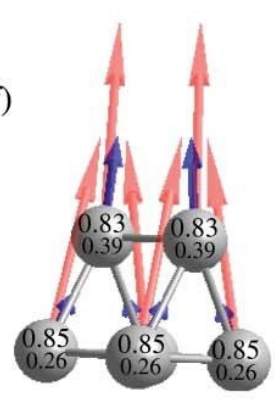

$\Delta E=49.75 \mathrm{meV} /$ atom

$m^{\prime} m^{\prime} 2$

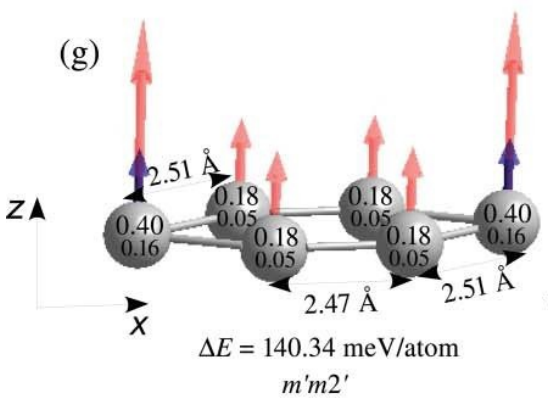

(h)

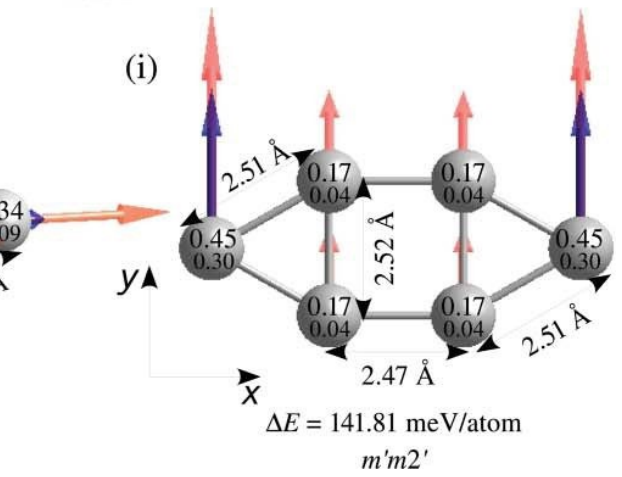

FIG. 8. Geometric and magnetic structures of a $\mathrm{Pt}_{6}$ cluster in different configurations. (a) and (b): Octahedral ground state geometry with the magnetic structure in the easy (a) and hard (b) axis configurations. (c) and (d): Capped square pyramid magnetized along the easy (c) and hard (d) directions. (e) and (f) Trigonal prismatic configuration magnetized along the easy (e) and hard (f) directions. (g)-(i): Planar structure with perpendicular (g) and in-plane [(h) and (i)] magnetizations. Below each configuration, the energy differences relative to the GS and the magnetic point group symmetry are listed. For the definition of symbols, see Fig. 1.

face-diagonals; pairs of moments are perpendicular to each other, see Fig. 8(a). The local-orbital moments are directed along the edges of the octahedron; moments aligned parallel to the same edge point into opposite directions. The atoms on the apical sites carry small spin and orbital moments which are also parallel to the equatorial plane. The local magnetic moments add up to a cluster moment of $M_{J}=0.83 \mu_{B}$, directed essentially perpendicular to one of the triangular facets of the octahedron. Due to the complex magnetic structure, the magnetic symmetry group is only $2^{\prime} 2^{\prime} 2$. The hard magnetic axis points along the fourfold symmetry axis of a slightly distorted octahedron with point group symmetry $4 \mathrm{~mm}$. The 
magnetic structure is collinear but breaks the fourfold symmetry; the magnetic point group symmetry is only $2^{\prime} 2^{\prime} 2$, as for the magnetic GS. Spin and orbital moments of the atoms in the equatorial plane have almost the same size as in the easy axis configuration but are rotated parallel to the overall magnetization direction. The total magnetic moment is $M_{J}=2.5 \mu_{B}$; the MAE is only $1.4 \mathrm{meV} /$ atom. The existence of a noncollinear magnetic GS of the octahedral $\mathrm{Pt}_{6}$ cluster might seem surprising, but the existence of competing exchange-interactions has been demonstrated already in the scalar relativistic calculations where an antiferromagnetic configuration was found to be favored over a nonmagnetic state for zero total spin and ferrimagnetic configurations were reported for low-moment isomers of the $\mathrm{Pt}_{6}$ octahedron.

The second structural isomer is a distorted square pyramid plus an ad-atom with a low symmetry (point group $m$ ). In the magnetic ground state the spin moments are aligned parallel to one of the edges of the basal plane of the pyramid and orbital moments are slightly canted relative to the spins [see Fig. 8(c)]. The local moments add to a cluster moment of $M_{J}=4.8 \mu_{B}$. A rotation of the magnetization direction to a perpendicular direction in the basal plane changes the cluster moment to $M_{J}=4.6 \mu_{B}$ (the spin moment is reduced from 3.5 to $3.2 \mu_{B}$, while the orbital moment increases by $0.1 \mu_{B}$ ) and costs an "in-plane" MAE of $2.9 \mathrm{meV} /$ atom [see Fig. 8(d)]. If the magnetic moment is perpendicular to the basal plane, both spin and orbital moments are reduced $\left(M_{S}=2.7 \mu_{B}, M_{L}=1.1 \mu_{B}, M_{J}=3.8 \mu_{B}\right)$. The perpendicular MAE is $12.5 \mathrm{meV} /$ atom.

The magnetic ground state for the trigonal prism in its high-moment state with $M_{J}=7.6 \mu_{B}$ is perpendicular to the triangular basis and the magnetic structure preserves the threefold symmetry (magnetic point group symmetry $\overline{6}^{\prime} \mathrm{m}^{\prime} 2^{\prime}$ ), see Fig. 8(e). If the magnetization is perpendicular to one of the rectangular facets, the slight differences in the local spin and orbital moments break the threefold symmetry and the magnetic point group symmetry is reduced to $\mathrm{m}^{\prime} \mathrm{m}^{\prime} 2$ [Fig. 8(f)]. Both spin and orbital moments are reduced, resulting in $M_{J}=6.9 \mu_{B}$. The MAE is $15.7 \mathrm{meV} /$ atom.

For the energetically least favorable planar configuration, we calculate an easy axis perpendicular to the plane of the cluster [see Fig. $8(\mathrm{~g})$ ]. The cluster is in a low-moment state with $M_{J}=2.0 \mu_{B}$. In-plane configurations with the magnetization oriented along the long and short axes of the cluster and a total magnetic moment of about $2 \mu_{B}$ [Figs. 8(h) and $8(\mathrm{i})$ ] are disfavored by MAEs of 0.63 and $0.82 \mathrm{meV} /$ atom, respectively. MAE and spin and orbital anisotropies are much smaller than those for the planar $\mathrm{Pt}_{5}$ cluster.

No AFM configurations could be found for any of the structural isomers of the $\mathrm{Pt}_{6}$ cluster. It is interesting that for both the $\mathrm{Pt}_{5}$ and $\mathrm{Pt}_{6}$ clusters, low-moment configurations are stabilized in the planar configurations while high-moment states are formed in the three-dimensional structures.

\section{DISCUSSION AND CONCLUSIONS}

In summary we find that spin-orbit coupling has a strong influence on the geometric and magnetic structures of small Pt clusters. (i) Planar structures are stabilized up to $\mathrm{Pt}_{5}$; for larger clusters, the energetic order of different structural isomers is changed. (ii) The clusters have large orbital magnetic moments comparable to the spin moments; both are strongly anisotropic. The anisotropy of the moments decreases with increasing size of the clusters. (iii) SOC leads to a mixing of different spin-states; the total magnetic moments can be enhanced as well as reduced compared to a scalar relativistic treatment. (iv) Local spin and orbital moments can be noncollinear, but the total spin and orbital moments of the cluster are always aligned. (v) An antiferromagnetic ground state is found for both planar and tetrahedral $\mathrm{Pt}_{4}$ clusters. For a planar $\mathrm{Pt}_{5}$ cluster the moments are ferromagnetically aligned, but the first excited magnetic state shows antiferromagnetic ordering. (vi) The magnetic potential-energy surface as a function of the orientation of the magnetization (or of the local magnetic moments for AFM states) consists of interpenetrating sheets representing different magnetic isomers (low- and high-moment FM as well as AFM states). (vii) The magnetic anisotropy energy (taken as the energy of the lowest saddle-point on the magnetic energy surface leading to magnetization reversal of an FM GS) can correspond to a lowmoment state $\left(\mathrm{Pt}_{2}, \mathrm{Pt}_{3}\right)$, an AFM isomer $\left(\mathrm{Pt}_{5}\right)$, or even to a configuration with higher total moment than that in the GS $\left(\mathrm{Pt}_{6}\right)$. No MAE can be given for $\mathrm{Pt}_{4}$ because the GS is antiferromagnetic.

These surprising results are a consequence of the strong spin-orbit coupling inducing a splitting of the eigenstates, which is comparable to the exchange splitting and much larger than the average level spacing. In the absence of exchange splitting and SOC, the geometric structure of the nonmagnetic clusters is determined by the formation and occupation of bonding/antibonding states, depending on the hybridization of the atomic orbitals. Formation of magnetic moments leads to an exchange splitting between majority and minority spin states. For a metal with a nearly filled d-band such as Pt, the majority states will be completely occupied whereas the occupation of the antibonding minority states will be reduced (favoring the formation of a magnetic GS). Even at a scalar relativistic level, magnetism can influence the structural stability of clusters. For example, for a $\mathrm{Pt}_{6}$ cluster with vanishing total moment, a square pyramid plus an ad-atom and an incomplete trigonal bipyramid are almost energetically degenerated and favored over the octahedron or the trigonal prism. ${ }^{7}$ Whether SOC leads to a further splitting and a reordering of the eigenstates around the Fermi level depends on the magnetic symmetry of the cluster. Without SOC both spin-up and spin-down states can be classified according to the point group symmetry of the cluster. For example, for a $\mathrm{Pt}_{2}$ dimer $d d \pi$ and $d d \delta$ states are twofold degenerate due to mirror and time-reversal symmetry. The degeneracy of these states is conserved for magnetization perpendicular to the dimer axis but lifted for parallel magnetization because reflection and time-reversal are no longer symmetry operations. The large SOC splitting changes the occupation of states close to the Fermi level and favors the occupation of states with a large orbital moment. ${ }^{10,12}$ For the trimer and pentamer the geometric structure corresponds to PGS $2 \mathrm{~mm}$. The magnetic PGS (Ref. 45) of the GS of $\mathrm{Pt}_{3}$ is $m^{\prime} m^{\prime} 2$ and that of the other two stationary configurations is $2^{\prime} m^{\prime} m$ (where the 
symbols $m^{\prime}$ and $2^{\prime}$ indicates that the symmetry operation must by combined with a time-reversal operation which reverses the direction of axial vectors such as the magnetic moment). For the $\mathrm{Pt}_{4}$ cluster the geometric PGS $\mathrm{mmm}$ is changed to $m^{\prime} m^{\prime} m^{\prime}$ for the AFM and to $m m^{\prime} m^{\prime}$ for the FM states. For the FM states of $\mathrm{Pt}_{5}$ with crystallographic point group $2 \mathrm{~mm}$, the time-reversal operation has to be applied to a mirror operation if the magnetization is parallel to the mirror plane and to the twofold rotation if the magnetization is perpendicular to the axis (as indicated in Fig. 4); similar rules apply to the AFM configurations. For the GS of the $\mathrm{Pt}_{6}$ cluster the crystallographic PGS $m \overline{3} m$ of the octahedron is reduced by different local magnetic moments on crystallographically equivalent sites to $2^{\prime} 2^{\prime} 2$. For the first excited state the geometric symmetry is reduced to $4 / \mathrm{mmm}$, but the magnetic symmetry is again $2^{\prime} 2^{\prime} 2$. Mirror and/or rotational symmetries induce degeneracies which are lifted by SOC if the symmetry is broken for a given direction of the magnetization. The stabilization of different magnetic isomers depends on the interplay between exchange splitting and spin-orbit splitting of eigenstates, depending on the magnitude of the exchange splitting (and hence of the magnetic moment) and on the direction of magnetization. These very general considerations also suggest that similar strong spin-orbit effects will also be found in other nanostructures of the heavy $5 \mathrm{~d}$ metals. An example has already been reported by Smogunov et al. ${ }^{15}$ who reported for a Pt monowire ferromagnetic order if the moment is perpendicular to the axis of the wire which disappears completely if the magnetization is forced to be parallel to the wire.

The structural and magnetic properties of the Pt clusters illustrate the complexity of the interplay of strong SOC, exchange splitting, and hybridization of the electronic eigenstates. It must be emphasized that a combined optimization of the geometric and magnetic degrees of freedom requires the absence of any symmetry constraints and extremely accurate calculations with very stringent limits on total energy and force convergence. We believe that the divergent results reported in the literature are largely due to insufficient convergence (which may indeed be very slow). It must also be emphasized that it will not be possible to test our predictions for the magnetic anisotropy experimentally because gas-phase clusters can always rotate freely such that the easy magnetic axis will be aligned with an applied magnetic field. However, these results are important for a fundamental understanding of the intrinsic properties of these clusters, and they form a reference for investigations of clusters supported on a solid surface where the orientation of the cluster is fixed by the binding to the substrate and where the magnetic anisotropy is determined by the interplay between the intrinsic anisotropy of the cluster and that imposed by the interaction with the substrate. First results for Pt clusters supported on graphene layers will be reported soon. ${ }^{48}$

${ }^{1}$ R. Wiesendanger, Rev. Mod. Phys. 81, 1495 (2009).

${ }^{2}$ D. Sellmyer and R. Skomski, Advanced Magnetic Nanostructures (Springer, New York, 2006).

${ }^{3}$ M. N. Huda, M. K. Niranjan, B. R. Sahu, and L. Kleinman, Phys. Rev. A 73, 053201 (2006).

${ }^{4}$ H. Häkkinen, B. Yoon, U. Landman, X. Li, H.-J. Zhai, and L.-S. Wang, J. Phys. Chem. A 107, 6168 (2003).
${ }^{5}$ S. Gilb, P. Weis, F. Furche, R. Ahlrichs, and M. M. Kappes, J. Chem. Phys. 116, 4094 (2002).

${ }^{6}$ F. Baletto and R. Ferrando, Rev. Mod. Phys. 77, 371 (2005)

${ }^{7}$ T. Futschek, M. Marsman, and J. Hafner, J. Phys.: Condens. Matter 17, 5927 (2005); J. Phys.: Condens. Matter 18, 9703 (2006).

${ }^{8}$ J. Kortus, T. Baruah, M. R. Pederson, C. Ashamn, and S. N. Khanna, Appl. Phys. Lett. 80, 4193 (2002).

${ }^{9}$ L. Fernandez-Seivane and J. Ferrer, Phys. Rev. Lett. 99, 183401 (2007); Phys. Rev. Lett. 101, 069903 (2008); see also supplementary material Document No. E-PRLTAO-99-018745 for detailed information on interatomic distances and local spin and orbital moments.

${ }^{10}$ T. O. Strandberg, C. M. Canali, and A. H. McDonald, Nature Mater. 6, 648 (2007); Phys. Rev. B 77, 174416 (2008).

${ }^{11}$ D. Fritsch, K. Koepernik, M. Richter, and H. Eschrig, J. Comput. Chem. 29, 2210 (2008)

${ }^{12}$ P. Błoński and J. Hafner, Phys. Rev. B 79, 224418 (2009).

${ }^{13}$ S. Sahoo, A. Hucht, M. E. Gruner, G. Rollmann, P. Entel, A. Postnikov, J. Ferrer, L. Fernández-Seivane, M. Richter, D. Fritsch, and S. Sil, Phys. Rev. B 82, 054418 (2010).

${ }^{14}$ Y. Mokrousov, G. Bihlmayer, S. Heinze, and S. Blügel, Phys. Rev. Lett. 96, 147201 (2006).

${ }^{15}$ A.Smogunov, A. Dal Corso, and E. Tosatti, Nat. Nanotechnol. 3, 22 (2008).

${ }^{16}$ J. Velev, R. F. Sabiryanov, S. S. Jaswal, and E. Y. Tsymbal, Phys. Rev. Lett. 94, 127203 (2005).

${ }^{17}$ P. Bruno, Phys. Rev. B 39, 865 (1989).

${ }^{18}$ C. M. Canali, A. Cehovin, and A. H. McDonald, Phys. Rev. Lett. 91, 046805 (2003).

${ }^{19}$ G. M. Pastor, J. Dorantes-Dávila, S. Pick, and H. Dreyssé, Phys. Rev. Lett. 75, 326 (1995).

${ }^{20}$ A. N. Andriotis and M. Menon, Phys. Rev. Lett. 93, 026402 (2004).

${ }^{21}$ G. Kresse and J. Hafner, Phys. Rev. B 47, 558 (1993); Phys. Rev. B 49, 14251 (1994)

${ }^{22}$ G. Kresse and D. Joubert, Phys. Rev. B 59, 1758 (1999).

${ }^{23}$ P. E. Blöchl, Phys. Rev. B 50, 17953 (1994).

${ }^{24}$ J. P. Perdew, J. A. Chevary, S. H. Vosko, K. A. Jackson, M. R. Pederson, D. J. Singh, and C. Fiolhais, Phys. Rev. B 46, 6671 (1992); Phys. Rev. B 48, 4978(E) (1993)

${ }^{25}$ S. H. Vosko, L. Wilk and M. Nusair, Can. J. Phys. 58, 1200 (1980)

${ }^{26}$ E. G. Moroni, G. Kresse, J. Hafner, and J. Furthmüller Phys. Rev. B 65 15629 (1997)

${ }^{27}$ J. Anton, T. Jacob, B. Fricke, and E. Engel, Phys. Rev. Lett. 89, 213001 (2002).

${ }^{28}$ G. Kresse and O. Lebacq, vasp Manual, http://cms.mpi.univie.ac.at/vasp/.

${ }^{29}$ L. Kleinman, Phys. Rev. B 21, 2630 (1980).

${ }^{30}$ A. H. MacDonald, W. E. Pickett, and D. D. Koelling, J. Phys. C 13, 2675 (1980).

${ }^{31}$ D. Hobbs, G. Kresse, and J. Hafner, Phys. Rev. B 62, 11556 (2000).

${ }^{32}$ M. Marsman and J. Hafner, Phys. Rev. B 66, 224409 (2002).

${ }^{33}$ G. H.O. Dalderoop, P. J. Kelly, and M. F. H. Schuurmans, Phys. Rev. B 50, 9989 (1994).

${ }^{34}$ E. Abata and M. Asdente, Phys. Rev. 140, A1303 (1965).

${ }^{35}$ L. Xiao and L. Wang, J. Phys. Chem A 108, 8605 (2004).

${ }^{36}$ A. Nie, J. Wu, C. Zhou, S. Yao, C. Luo, R. C. Forrey, and H. Cheng, Int. J. Quantum Chem. 107, 219 (2006).

${ }^{37}$ K. Bhattacharya and C. Majumder, Chem. Phys. Lett. 446, 374 (2007).

${ }^{38}$ A. Sebetci, Phys. Chem. Chem. Phys. 11, 921 (2009).

${ }^{39}$ J. M. Soler, E. Artacho, J. D. Gale, A. García, J. Junquera, P. Ordejón, and D. Sánchez-Portal, J. Phys.: Condens. Matter 14, 2745 (2002).

${ }^{40}$ N. Troullier and J. M. Martins, Phys. Rev. B 43, 1993 (1991).

${ }^{41}$ L. Fernández-Seivane, M. A. Oliveira, S. Sanvito, and J. Ferrer, J. Phys.: Condens. Matter 18, 7999 (2006).

${ }^{42}$ M. M. Airola and M. D. Morse, J. Chem. Phys. 113, 1313 (2002).

${ }^{43}$ A. Sebetci, Chem. Phys. 331, 9 (2006).

${ }^{44}$ X. Lin, N. J. Ramer, A. M. Rappe, K. C. Hass, W. F. Schneider, and B. L. Trout, J. Phys. Chem. 105, 7739 (2001).

${ }^{45}$ M. de Graef, Metall. Mater. Trans. A 41, 1321 (2010).

${ }^{46}$ D. Majumdar, D. Dai, and K. Balasubramanian, J. Chem. Phys. 113, 7919 (2000).

${ }^{47}$ D. Dai and K. Balasubramanian, J. Chem. Phys. 102, 648 (1995)

${ }^{48} \mathrm{P}$. Błoński and J. Hafner, "Geometric and magnetic properties of Pt clusters supported on graphene: relativistic density-functional calculations," to be published. 